\title{
The Uncertain Unit Root in Real GNP
}

\author{
By Glenn D. Rudebusch*
}

The impulse and propagation mechanisms of business cycles have long been debated; however, until recently, economists were in fairly broad agreement that business fluctuations could be studied separately from the secular growth of the economy. This separation was justified because, to a first approximation, the factors underlying trend growth were assumed to be stable at business-cycle frequencies. Indeed, the common practice of macroeconomists of all theoretical persuasions was to model movements in real GNP as stationary fluctuations around a linear deterministic trend (e.g., Finn Kydland and Edward C. Prescott, 1980; Olivier J. Blanchard, 1981). Such a trendstationary (TS) model of real GNP was the canonical empirical representation of aggregate output until the early 1980's.

In contrast to previous work, much of the research of the last ten years has assumed a unit root in the autoregressive representation of real GNP, which is inconsistent with a TS model of output. A model with a unit root, commonly termed a "differencestationary" (DS) model, implies that any stochastic shock to output contains an element that represents a permanent shift in the level of the series. If real GNP is best represented by a DS model, the traditional separation between business cycles and trend growth is incorrect. In the usual empirical versions of the DS model estimated for real GNP, output behaves more like a random walk than like transitory deviations

\footnotetext{
* Board of Governors of the Federal Reserve System, Division of Monetary Affairs, Washington, DC 20551. I thank two anonymous referees for comments as well as Bill Bell, Frank Diebold, Steve Durlauf, Spence Krane, Doug Steigerwald, and David Wilcox. Michelle Phillips and Roberto Sella provided research assistance. The views expressed are my own and are not necessarily shared by the Board of Governors or its staff.
}

from a steadily growing trend (e.g., John Y. Campbell and N. Gregory Mankiw, 1987; Peter K. Clark, 1987). The long-run trend of these DS representations is not fixed, as in a TS model, but stochastic; indeed, in the typical DS model, almost all fluctuations in output represent permanent shifts in trend rather than transitory movements in cycle. From another perspective, the essential difference between the two models can be found in the persistence of their dynamic responses to random shocks. In the DS model of output, the effect of a shock persists forever because the disturbance changes the trend component and thus affects the level of output in all future periods. In contrast, the impact of a shock in the TS model is transitory and is eliminated quite quickly as output reverts to its steady trend.

The widespread acceptance of a DS model for aggregate output was based on evidence that the hypothesis of a unit root in real GNP could not be rejected. ${ }^{1}$ Although this evidence was extremely robust across various data samples and unit-root testing procedures (see e.g., Charles R. Nelson and Charles I. Plosser, 1982; René M. Stulz and Walter Wasserfallen, 1985; James H. Stock and Mark W. Watson, 1986; Pierre Perron and Peter C. B. Phillips, 1987; Perron, 1988; George W. Evans, 1989), the distinct contrast between traditional TS models of output and recent DS models led many researchers to challenge the unit-root tests. In particular, some questioned the power of these tests, that is, their ability to reject the unit-root null hypothesis when it is indeed

\footnotetext{
${ }^{1}$ This acceptance was not generally shared by government and business economists. For example, the official series on potential output, which is used in policy and budget deliberations, has remained a smooth nonstochastic trend.
} 
false. $^{2}$ However, the indictment of low power against unit-root tests has not been a decisive criticism. The fact that unit-root tests may have low power against certain TS alternatives does not necessarily compromise the results from those tests. It would not be at all surprising if unit-root tests had little power against TS alternatives that mimicked a DS model and reverted to trend extremely slowly; all statistical tests have low power against alternatives that are "local" to the null. The failure to reject nearunit-root TS alternatives is of little economic importance, however, because these alternatives are indistinguishable in economic terms from the DS null over the time horizons of practical macroeconomic interest (say, shorter than 10 years). Critics of unit-root tests must instead make the stronger claim that unit-root tests have low power against plausible TS alternatives that display substantially different macroeconomic behavior than a plausible DS null model. Simple power studies are not well suited to answering this question.

This note addresses the argument that unit-root tests have low power only against local alternatives. The goal is to select the most plausible DS and TS representations for output, determine whether these representations have different short-run persistence properties, and then examine whether unit-root tests can distinguish between these models. In the next section, I examine the ordinary least-squares (OLS) estimates of a TS model and a DS model for the postwar sample of real GNP. These models are plausible representations of the data generating process and yet imply very different economic dynamics at the horizons of economic relevance. In Section II, I examine simulated data from these models and show that a unit-root test cannot distinguish be-

\footnotetext{
${ }^{2}$ Among many others, Bennett T. McCallum (1986), Francis X. Diebold and Rudebusch (1991), and David N. DeJong et al. (1992) have warned of the low power of unit-root tests. Other challenges have been made by DeJong and Whiteman (1991) from a Bayesian perspective and by Perron (1989) in a framework with structural breaks.
}

tween these particular TS and DS representations. Thus, the unit-root test has low power against a plausible TS model that is not local in economic terms to a plausible DS model. Section III extends the basic argument to account for small-sample bias in the estimated model coefficients.

\section{DS and TS Models of Real GNP}

Two obvious candidates for plausible TS and DS representations of the data generating process for real GNP are simply the OLS estimates of these models from the available data sample. The data consist of quarterly observations on U.S. postwar log real GNP per capita (denoted $Y_{t}$ ) from 1948:3 to 1988:4. Assuming second-order dependence, ${ }^{3}$ the sample OLS estimate of the TS model of aggregate output with a linear deterministic trend is

$$
\begin{aligned}
Y_{t}= & -\underset{ }{(0.321}+0.00)(0.00010) \\
& (0.073) \\
& -0.401 Y_{t-2}+u_{t} \quad \hat{\sigma}_{u}=0.01013 \\
& (0.073)
\end{aligned}
$$

(standard errors of the coefficients appear in parentheses). ${ }^{4} \mathrm{I}$ will refer to this specific model estimate for the sample as the $\mathrm{TS}_{\mathrm{OLS}}$ model.

Under the assumption of a unit root, the DS model for this data sample is estimated in first differences as

$$
\begin{array}{r}
\Delta Y_{t}=\underset{(0.001)}{0.003}+\underset{(0.074)}{0.369 \Delta Y_{t-1}+\hat{v}_{t}} \\
\qquad \hat{\sigma}_{v}=0.01035
\end{array}
$$

This particular sample DS model will be denoted as the $\mathrm{DS}_{\mathrm{OLS}}$ model.

\footnotetext{
${ }^{3}$ The analysis was repeated assuming that the order of the model was four, six, and eight. Similar persistence and power results were obtained.

${ }^{4}$ The last two quarters of 1948 were used as fixed initial conditions.
} 
The estimated models (1) and (2) both appear to fit real GNP per capita fairly well; the standard deviations of their residuals are quite close, and plots of the residuals suggest no obvious outliers. In addition, $Q$ statistics computed from the fitted residuals provide little evidence against the null hypothesis of no serial correlation at a variety of lags.

However, the estimated $\mathrm{TS}_{\mathrm{OLS}}$ and $\mathrm{DS}_{\mathrm{OLS}}$ models have very different implications for the persistence of the dynamic response of output to a random disturbance. To measure this persistence, consider the movingaverage representation for the first difference of output implied by a TS or DS model:

$$
\Delta Y_{t}=k+\varepsilon_{t}+a_{1} \varepsilon_{t-1}+a_{2} \varepsilon_{t-2}+\ldots
$$

where $k$ is some constant and $\varepsilon_{t}$ is the innovation of the model. In this form, the sum of the $a_{i}$ 's measures the model response to a unit innovation. ${ }^{5} \mathrm{~A}$ unit shock in period $t$ affects $\Delta Y_{t+h}$ by $a_{h}$ and affects $Y_{t+h}$ by $c_{h} \equiv 1+a_{1}+\cdots+a_{h}$. Thus, for various horizons, the cumulative response $c_{h}$ answers the question: how does a shock today affect the level of real output in the short, medium, and long run? With quarterly data, for example, $c_{20}$ measures the impact of a shock today on $Y_{t}$ five years hence.

In the limit, the effect of a unit shock today on the level of output infinitely far in the future is given by $c_{\infty}$. For any TS series, $c_{\infty}=0$, because the effect of any shock is eliminated as reversion to the deterministic trend eventually dominates. For a DS series, $c_{\infty} \neq 0$; that is, each shock has some permanent effect. However, the impulse response of real output at an infinite horizon is of no practical economic significance; indeed, horizons of less than 10 years are usually of greatest interest. At these short horizons, the dynamic responses of TS and

\footnotetext{
${ }^{5}$ This measure of persistence is described further in Campbell and Mankiw (1987) and Diebold and Rudebusch (1989).
}

DS models may be quite similar or quite different depending on the values taken by the parameters of the models. Thus, the presence of a unit root determines whether $c_{\infty}$ is positive or zero, but it does not determine all of the model properties of economic interest. It is in this sense that, as noted in the introduction, focusing solely on the existence of unit roots and on the power of unit-root tests against arbitrary TS alternatives is insufficient. What is of economic relevance is the ability of unit-root tests to recognize when data have been generated from TS models that differ substantially from the DS model at short horizons (i.e., the ability to identify economically nonlocal alternatives). Consequently, a comparison of the persistence properties of the estimated $\mathrm{TS}_{\mathrm{OLS}}$ and $\mathrm{DS}_{\mathrm{OLS}}$ models at relevant horizons is required.

The estimated model responses are shown in Table 1, with standard errors in parentheses. ${ }^{6}$ The impulse response of the $\mathrm{DS}_{\mathrm{OLS}}$ model implies not only shock persistence but shock magnification. The effect of an innovation is not reversed through time, and it eventually increases the level of real GNP by more than one and a half times the size of the innovation $\left(c_{20}=1.59\right)$. In contrast, the TS model exhibits fairly rapid reversion to trend, with 85 percent of a shock dissipated after five years $\left(c_{20}=0.15\right)$. Thus, the cumulative impulse responses of these two models, each estimated from the same data sample, imply very different economic dynamics at cyclical frequencies. Because the $\mathrm{TS}_{\mathrm{OLS}}$ and $\mathrm{DS}_{\mathrm{OLS}}$ models of aggregate output have such different persistence properties, it would be useful to have a test capable of distinguishing between them. The next section explores the ability of one commonly used unit-root test to accomplish this task.

\footnotetext{
${ }^{6}$ These standard errors are calculated as follows. Let the cumulative impulse response at horizon $h$ be given by $c_{h}=F\left(\rho_{1}, \ldots, \rho_{k}\right)$, and let $\mathbf{f}$ denote the vector of partials of $F$ with respect to the parameters; then, the standard error equals $\sqrt{\mathbf{f}^{\prime} \boldsymbol{\Sigma} \mathbf{f}}$, where $\boldsymbol{\Sigma}$ is the estimated variance-covariance matrix of the autoregressive parameters. The standard errors account for parameter uncertainty but not for unit-root uncertainty.
} 
Table 1-Cumulative Impulse Responses of OLS Models

\begin{tabular}{|c|c|c|c|c|c|c|c|c|c|}
\hline \multirow[b]{2}{*}{ Model } & \multicolumn{9}{|c|}{ Horizon (quarters) } \\
\hline & 1 & 2 & 4 & 8 & 12 & 16 & 20 & 30 & 40 \\
\hline $\mathrm{DS}_{\mathrm{OLS}}$ & $\begin{array}{c}1.37 \\
(0.07)\end{array}$ & $\begin{array}{c}1.51 \\
(0.13)\end{array}$ & $\begin{array}{c}1.57 \\
(0.17)\end{array}$ & $\begin{array}{c}1.59 \\
(0.19)\end{array}$ & $\begin{array}{c}1.59 \\
(0.19)\end{array}$ & $\begin{array}{c}1.59 \\
(0.19)\end{array}$ & $\begin{array}{c}1.59 \\
(0.19)\end{array}$ & $\begin{array}{c}1.59 \\
(0.19)\end{array}$ & $\begin{array}{c}1.59 \\
(0.19)\end{array}$ \\
\hline $\mathrm{TS}_{\mathrm{OLS}}$ & $\begin{array}{c}1.33 \\
(0.07)\end{array}$ & $\begin{array}{c}1.38 \\
(0.13)\end{array}$ & $\begin{array}{c}1.19 \\
(0.18)\end{array}$ & $\begin{array}{c}0.73 \\
(0.23)\end{array}$ & $\begin{array}{c}0.43 \\
(0.23)\end{array}$ & $\begin{array}{c}0.25 \\
(0.20)\end{array}$ & $\begin{array}{c}0.15 \\
(0.15)\end{array}$ & $\begin{array}{c}0.04 \\
(0.07)\end{array}$ & $\begin{array}{c}0.01 \\
(0.02)\end{array}$ \\
\hline
\end{tabular}

Note: Standard errors are given in parentheses.

\section{Application of a Unit-Root Test}

The augmented Dickey-Fuller unit-root test (David A. Dickey and Wayne F. Fuller, 1981 ) is often used to try to distinguish a TS model from a DS model. ${ }^{7}$ For the secondorder models under consideration, the augmented Dickey-Fuller regression takes the following form:

$$
Y_{t}=\hat{\mu}+\hat{\gamma} t+\hat{\delta} Y_{t-1}+\hat{\phi}_{1} \Delta Y_{t-1}+\hat{\varepsilon}_{t}
$$

Under the unit-root (or DS model) null hypothesis, $\delta=1$; thus, the Dickey-Fuller test statistic is simply the $t$ test, $\hat{\tau} \equiv(\hat{\delta}-1) /$ $\operatorname{SE}(\hat{\delta})$, where $\operatorname{SE}(\hat{\delta})$ is the standard error of the estimated coefficient.

For the postwar real GNP data under consideration, the sample value of the Dickey-Fuller test, which is denoted as $\hat{\tau}_{\text {samp }}$, is equal to -2.98 . However, this statistic does not have the usual Student- $t$ distribution, but is skewed toward negative values. At the 10-percent significance level, Dickey and Fuller (1981) calculate the appropriate asymptotic critical value to be -3.12 . Thus, the evidence from this sample, in accordance with the findings of previous researchers, suggests that the DS model for real GNP cannot be rejected at even the 10 -percent level.

\footnotetext{
${ }^{7}$ Similar results to those below were also obtained with the Dickey-Fuller normalized-bias and likelihood-ratio tests, as well as with the generalized Phillips test (Phillips and Perron, 1988).
}

However, the critical values provided by Dickey and Fuller (1981) for their augmented test are only valid asymptotically. In finite samples, the distribution of $\hat{\tau}$ will usually depend on the sample size and nuisance-parameter values (see e.g., Gene Evans and Savin, 1984). These factors can be taken into account by examining simulated data from the $\mathrm{DS}_{\mathrm{OLS}}$ model and calculating the exact probability of obtaining the sample value of the test statistic from this particular null model. This ensures correct size for the test. More importantly, however, by simulating the $\mathrm{TS}_{\mathrm{OLS}}$ model, the exact probability of obtaining $\hat{\tau}_{\text {samp }}$ from this particular alternative model can also be obtained. This allows correct assessment of test power against what is arguably one of the most interesting alternatives.

The test-statistic probability distributions conditional on the OLS models are exhibited in Figure 1. The distribution of $\hat{\tau}$ conditional on the $\mathrm{DS}_{\mathrm{OLS}}$ model is denoted $f_{\mathrm{DS}}(\hat{\tau})$, while the distribution of $\hat{\tau}$ conditional on the $\mathrm{TS}_{\mathrm{OLS}}$ model is denoted $f_{\mathrm{TS}}(\hat{\tau})$. Each distribution is formed from 10,000 realizations of the test statistic calculated from 10,000 simulated data samples generated from the particular model. ${ }^{8}$ The actual sample value of the test statistic $\left(\hat{\tau}_{\text {samp }}=-2.98\right)$ is shown as a vertical dotted line.

\footnotetext{
${ }^{8}$ The samples are generated with normal independently and identically distributed errors with sample size and initial conditions that matched those in equations (1) and (2).
} 


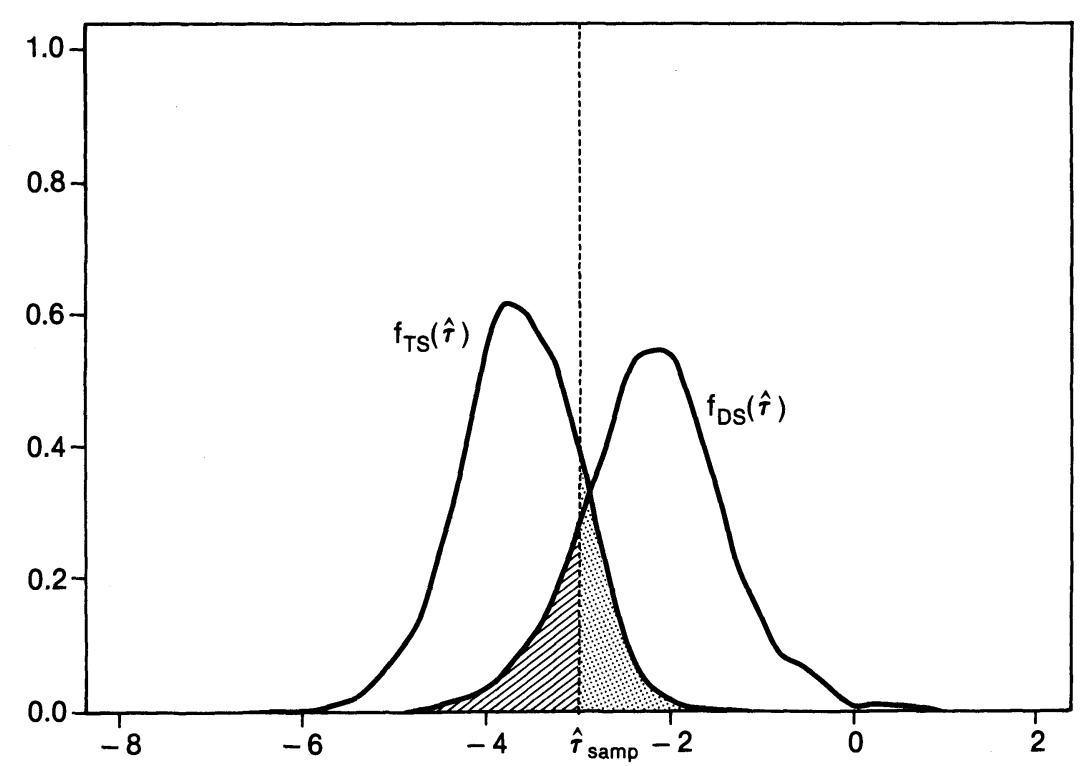

Figure 1. Empirical Densities of $\hat{\tau}$ From $\mathrm{DS}_{\text {Ols }}$ and TS $\mathrm{Tl}_{\text {OlS }}$ Models

There are two areas in Figure 1 of special interest. The hatched area under $f_{\mathrm{DS}}(\hat{\tau})$ and to the left of $\hat{\tau}_{\text {samp }}$ represents the probability of obtaining a value of the $t$ test equal to or smaller than -2.98 , conditional on the DS model of equation (2). This $p$ value, is denoted as

$\mathrm{DS}_{\mathrm{OLS}} p$ value

$$
\equiv \operatorname{prob}\left(\hat{\tau} \leq \hat{\tau}_{\text {samp }} \mid \mathrm{DS}_{\text {OLS }} \text { model }\right)
$$

and represents the marginal significance level for rejection of the null hypothesis for the $\mathrm{DS}_{\mathrm{OLS}}$ model. This probability equals 0.15 ; that is, given the sample test statistic, one could not reject the DS model at anything less than the 15-percent level in a classical hypothesis test. This is consistent with the usual inability to reject the DS model for real GNP at conventional significance levels.

The other area of interest is the shaded region under $f_{\mathrm{TS}}(\hat{\tau})$ and to the right of $\hat{\tau}_{\text {samp. }}$. This area represents the probability of obtaining a value of the $t$ test equal to or greater than -2.98 , conditional on the TS model of equation (1). This probability is denoted as

$\mathrm{TS}_{\mathrm{OLS}} p$ value

$$
\equiv \operatorname{prob}\left(\hat{\tau} \geq \hat{\tau}_{\text {samp }} \mid \mathrm{TS}_{\mathrm{OLS}} \text { model }\right) .
$$

For real GNP, the $\mathrm{TS}_{\mathrm{OLS}} p$ value is 0.22 , so one would not be able to reject the estimated $\mathrm{TS}_{\mathrm{OLS}}$ model at even the 20 -percent significance level. ${ }^{9}$

In short, the sample statistic for the augmented Dickey-Fuller test does not provide strong evidence against either the estimated $\mathrm{DS}_{\text {OLS }}$ model or the $\mathrm{TS}_{\mathrm{OLS}}$ model for real GNP. Earlier papers that are unable to reject a unit root in output provide only one side of the relevant evidence for inference regarding the DS model. The other side, namely, the inability to reject a plausible TS model that exhibits transitory cyclical dynamics of a traditional nature, is at least as convincing.

${ }^{9}$ An equivalent statement of this result is that the $\hat{\tau}$ test at the 15-percent significance level has power against the $\mathrm{TS}_{\mathrm{OLS}}$ alternative of only 78 percent. 
Table 2-Properties of the OLS Estimate of an AR(1) Model

\begin{tabular}{lccccccc}
\hline \hline & \multicolumn{7}{c}{$\rho_{1}$} \\
\cline { 2 - 8 } Statistic & 0.40 & 0.60 & 0.80 & 0.85 & 0.90 & 0.95 & 1.00 \\
\hline$E\left(\hat{\rho}_{1}\right)$ & 0.38 & 0.57 & 0.76 & 0.81 & 0.86 & 0.90 & 0.94 \\
$\operatorname{prob}\left(\hat{\rho}_{1}<\rho_{1}\right)$ & 0.61 & 0.65 & 0.72 & 0.76 & 0.81 & 0.89 & 0.99 \\
\hline
\end{tabular}

Note: Each column is based on 10,000 samples (each with 160 observations) drawn from an $\operatorname{AR}(1)$ with an autoregressive coefficient equal to $\rho_{1}$.

\section{Unbiased DS and TS Models}

At first glance, the $\mathrm{DS}_{\mathrm{OLS}}$ and $\mathrm{TS}_{\mathrm{OLS}}$ models might appear to be the most plausible candidates for DS and TS representations of the data generating process of real GNP. However, although the OLS estimates of these autoregressive models are consistent and asymptotically normal, they are biased in small samples because the presence of lagged dependent variables violates the assumption of nonstochastic regressors in the classical linear regression model. This bias is easy to illustrate for the OLS estimate of the autoregressive parameter of an $\mathrm{AR}(1)$ process,

$$
x_{t}=\mu+\gamma t+\rho_{1} x_{t-1}+\varepsilon_{t} .
$$

Based on 10,000 samples of size 160 generated from equation (5), Table 2 provides the mean value of the OLS estimate, $\hat{\rho}_{1}$, as well as the proportion of estimates that are less than the true value of $\rho_{1} \cdot{ }^{10}$ For example, if the true $\rho_{1}$ is equal to 0.95 , the mean OLS estimate is 0.90 , and 89 percent of the estimates are less than 0.95 . The size of the autoregressive parameter bias that pushes the average OLS estimate below its true value varies with the value of the true parameter, but it is most severe for near-unitroot models (i.e., those with $\rho$ close to 1.0).

A significant bias in the OLS sample estimates is potentially a serious shortcoming of the simulation methodology pursued in the previous two sections. In particular, the es-

\footnotetext{
${ }^{10}$ Table 2 is generated with $\mu=\gamma=x_{0}=0$ and disturbances drawn from a standard normal distribution.
}

timation bias implies that the sample OLS models used above probably understate the actual amount of persistence in real output. Suppose, for example, that an AR(1) TS representation like equation (5) was fit to real output, and the resulting OLS sample estimate $\hat{\rho}_{1}$ was equal to 0.90 ; consequently, the associated estimate of the 10-period cumulative impulse response, $\hat{c}_{10}$, would be 0.34 . Assuming that the OLS estimate was equal to its mean, $E\left(\hat{\rho}_{1}\right)$, then the true parameter would be 0.95 , and the actual value of $c_{10}$ would be 0.60 . Thus, in this case, the OLS sample estimate, on average, understates the amount of persistence.

Arguably, more plausible candidates than the $\mathrm{TS}_{\mathrm{OLS}}$ and $\mathrm{DS}_{\mathrm{OLS}}$ models for the datagenerating process would correct the parameters for small-sample bias. ${ }^{11}$ First-order approximate bias corrections can be calculated for both the TS and DS models quite easily. The bias of the $\mathrm{DS}_{\mathrm{OLS}}$ model, which is an AR(1) model with an unknown drift parameter, is treated in F. H. C. Marriott and J. A. Pope (1954). They show that, ignoring second-order terms, the expected value of the OLS estimate $\hat{\rho}_{1}$ is given by

$$
E\left(\hat{\rho}_{1}\right)=\rho_{1}-\left(1+3 \rho_{1}\right) / T
$$

where $T$ is the sample size. Substituting the sample OLS estimate for its expected value

\footnotetext{
${ }^{11}$ In this paper, I consider approximate meanunbiased estimators of the TS and DS models. In Rudebusch (1992), I examine median-unbiased estimators obtained through repeated simulations, a procedure which would lead to qualitatively similar results if applied to the data set in this paper.
} 
provides a bias-corrected estimator

$$
\tilde{\rho}_{1}=\left(T \hat{\rho}_{1}+1\right) /(T-3) .
$$

Note that, to a first-order approximation, this estimator is unbiased: ${ }^{12}$

$$
E\left(\tilde{\rho}_{1}\right)=\rho_{1} .
$$

Applying (7) to the $\mathrm{DS}_{\mathrm{OLS}}$ model for postwar real GNP given in (2), where $\hat{\rho}_{1}=0.369$, I calculate the autoregressive coefficient of the bias-corrected DS model (denoted as the $\mathrm{DS}_{\mathrm{BC}}$ model) to be $\tilde{\rho}_{1}=0.383$.

Similarly, for the $\mathrm{TS}_{\text {OLS }}$ model, which is an $\operatorname{AR}(2)$ with linear trend, the (first-order) bias in the estimated parameters can be determined using the results in Robert A. Stine and Paul Shaman (1989). Correcting for bias gives a $\mathrm{TS}_{\mathrm{BC}}$ model with autoregressive coefficients:

$$
\tilde{\rho}_{1}=\left(T \hat{\rho}_{1}+2+2 \tilde{\rho}_{2}\right) /(T-1)
$$

for the one-period lag, and

$$
\tilde{\rho}_{2}=\left(T \hat{\rho}_{2}+3\right) /(T-5)
$$

for the two-period lag. For postwar real GNP, the $\mathrm{TS}_{\mathrm{BC}}$ model has autoregressive coefficients $\tilde{\rho}_{1}=1.351$ and $\tilde{\rho}_{2}=-0.395$.

The bias-corrected $\mathrm{DS}_{\mathrm{BC}}$ and $\mathrm{TS}_{\mathrm{BC}}$ models both display somewhat greater persistence than the $\mathrm{DS}_{\mathrm{OLS}}$ and $\mathrm{TS}_{\mathrm{OLS}}$ models. The bias correction embodied in the $\mathrm{DS}_{\mathrm{BC}}$ model is in the same direction and of the same magnitude as the one suggested by Table 2. In particular, because the root of the associated lag-operator polynomial is so far from the unit circle, the bias of the $\mathrm{DS}_{\text {OLs }}$ model is quite modest. ${ }^{13}$ For the

\footnotetext{
${ }^{12}$ Of course, reduced bias does not necessarily ensure that $\tilde{\rho}_{1}$ is a better estimator. However, Guy $\mathrm{H}$. Orcutt and Herbert S. Winokur (1969) explore the properties of this estimator through simulations and find that it often has a smaller mean squared error than the OLS estimator. Theoretical results on this issue are provided in Hong-Ching Zhang (1989).

${ }^{13}$ The bias in the DS ${ }_{\text {OLS }}$ model is even smaller than the one in Table 2 because a linear trend is not estimated. With a linear trend, as in Table 2, the bias is given by $E\left(\hat{\rho}_{1}\right)-\rho_{1}=-\left(2+4 \rho_{1}\right) / T$.
}

second-order $\mathrm{TS}_{\mathrm{OLS}}$ and $\mathrm{TS}_{\mathrm{BC}}$ models, a useful metric with which to judge their closeness to a nonstationary model is simply the sum of the autoregressive coefficients (see e.g., Phillips, 1991). The sum of the OLS estimates $\hat{\rho}_{1}$ and $\hat{\rho}_{2}$ equals 0.933 , while the sum of the bias-corrected coefficients $\tilde{\rho}_{1}$ and $\tilde{\rho}_{2}$ equals $0.955-$ a clear, though somewhat small, shift toward nonstationarity. ${ }^{14}$

More specifically, the implications of the bias correction for judging the persistence of real GNP are given in Table 3 , which contrasts the impulse responses of the $\mathrm{DS}_{\mathrm{BC}}$ and $\mathrm{TS}_{\mathrm{BC}}$ models. The impulse response of the $\mathrm{DS}_{\mathrm{BC}}$ model implies a shock persistence that is virtually indistinguishable from that of the $\mathrm{DS}_{\mathrm{OLS}}$ model, which is not surprising given the trivial size of the coefficient bias. Moreover, the $\mathrm{TS}_{\mathrm{BC}}$ model exhibits reversion to trend only slightly less rapid than that of the $\mathrm{TS}_{\mathrm{OLS}}$ model. For the $\mathrm{TS}_{\mathrm{BC}}$ model, almost two-thirds of a shock is dissipated after five years $\left(c_{20}=0.37\right)$. Most importantly, it remains true that the $\mathrm{DS}_{\mathrm{BC}}$ and $\mathrm{TS}_{\mathrm{BC}}$ candidates for plausible representations of aggregate output have quite different implications about dynamic responses over fairly short horizons.

As a final step, one can ask whether the augmented Dickey-Fuller test can distinguish between these two models. Based on 10,000 samples generated from the $\mathrm{DS}_{\mathrm{BC}}$ model, ${ }^{15}$ I obtain the probability of the sample test statistic as

$\mathrm{DS}_{\mathrm{BC}} p$ value

$$
\equiv \operatorname{prob}\left(\hat{\tau} \leq \hat{\tau}_{\text {samp }} \mid \mathrm{DS}_{\mathrm{BC}} \operatorname{model}\right)=0.15 .
$$

In contrast, the probability of the test statistic under the bias-corrected alternative

\footnotetext{
${ }^{14}$ The bias correction in the AR(2) TS model may appear to be surprisingly small in light of the large biases shown for the AR(1) model in Table 2. However, as noted by Stine and Shaman (1989), the results for the AR(1) model do not generalize to higher-order models. Indeed, there are cases in which the bias (to a first-order approximation) moves the roots of the lagoperator polynomial closer to the unit circle.

${ }^{15}$ The OLS estimates of the trend and intercept are used in generating data.
} 
Table 3-Cumulative Impulse Responses of Bias-Corrected Models

\begin{tabular}{lccccccccc}
\hline \hline & \multicolumn{8}{c}{ Horizon (quarters) } \\
\cline { 2 - 10 } Model & 1 & 2 & 4 & 8 & 12 & 16 & 20 & 30 & 40 \\
\hline DS $_{\mathrm{BC}}$ & 1.38 & 1.53 & 1.61 & 1.62 & 1.62 & 1.62 & 1.62 & 1.62 & 1.62 \\
$\mathrm{TS}_{\mathrm{BC}}$ & 1.35 & 1.43 & 1.32 & 0.97 & 0.70 & 0.51 & 0.37 & 0.16 & 0.07 \\
\hline
\end{tabular}

model is

$$
\begin{aligned}
& \mathrm{TS}_{\mathrm{BC}} p \text { value } \\
& \quad \equiv \operatorname{prob}\left(\hat{\tau} \geq \hat{\tau}_{\text {samp }} \mid \mathrm{TS}_{\mathrm{BC}} \text { model }\right)=0.24 .
\end{aligned}
$$

These probabilities provide further confirmation of the inability of unit-root tests to identify plausible TS alternative models for real output that display low persistence. In sum, the biases present in the OLS estimates are not substantial enough to change the conclusions from Section I and II.

\section{Conclusion}

Based on the usual unit-root tests, little can be said about the relative likelihood of the specific DS and TS models of real GNP given above. In particular, plausible TS alternatives that fit the data cannot be detected because of low test power. The appropriate conclusion from unit-root tests on this data sample is that the existence of a unit root is uncertain.

Furthermore, the unit-root tests employed display low power against plausible TS models that display quite different economic behavior than a plausible DS null model. If the TS models described above correctly portray the persistence of real GNP, then the DS model does not provide a good approximation of the dynamic response of output at even short-run cyclical horizons of, say, five years.

In light of the impulse responses of the TS models, the appropriate confidence intervals for estimates of short-term or medium-term persistence are much larger than ones given conditional on the existence of a unit root. This suggests the importance of measuring the confidence intervals for estimates of persistence without conditioning on the TS or DS model. In Diebold and
Rudebusch (1989), approximate estimates of such intervals are obtained by using a model of fractional integration that nests the TS and DS models. Stock (1991) also provides a step in this direction by obtaining confidence intervals for the largest autoregressive root.

In sum, the evidence in this paper and in other recent work, notably Lawrence $J$. Christiano and Martin Eichenbaum (1990), suggests that a new consensus should be formed that stresses the uncertainty about the existence of a unit root in real output and the uncertainty about the amount of persistence of macroeconomic shocks.

\section{REFERENCES}

Blanchard, Olivier J., "What is Left of the Multiplier Accelerator?" American Economic Review, May 1981 (Papers and Proceedings), 71, 150-4.

Campbell, John Y. and Mankiw, N. Gregory, "Are Output Fluctuations Transitory?" Quarterly Journal of Economics, November 1987, 102, 857-80.

Christiano, Lawrence J. and Eichenbaum, Martin, "Unit Roots in Real GNP: Do We Know, and Do We Care?" CarnegieRochester Conference Series on Public Policy, Spring 1990, 32, 7-62.

Clark, Peter K., "The Cyclical Component of U.S. Economic Activity," Quarterly Journal of Economics, November 1987, 102, 797-814.

DeJong, David N., Nankervis, John C., Savin, N. E. and Whiteman, Charles H., "Integration versus Trend Stationarity in Time Series," Econometrica, March 1992, 60, 423-33. and Whiteman, Charles H., "Reconsidering "Trends and Random Walks in Macroeconomic Time Series'," Journal of Monetary Economics, October 1991, 28, 
221-54.

Dickey, David A. and Fuller, Wayne F., "Likelihood Ratio Statistics for Autoregressive Time Series with a Unit Root," Econometrica, July 1981, 49, 1057-72.

Diebold, Francis X. and Rudebusch, Glenn D., "Long Memory and Persistence in Aggregate Output," Journal of Monetary Economics, September 1989, 24, 189-209. and "On the Power of Dickey-Fuller Tests Against Fractional Alternatives," Economics Letters, 1991, 35 (2), 155-60.

Evans, Gene and Savin, N. E., "Testing for Unit Roots II," Econometrica September 1984, 52, 1241-70.

Evans, George W., "Output and Unemployment Dynamics in the United States: 1950-1985," Journal of Applied Econometrics, July-September 1989, 4, 213-37.

Kydland, Finn and Prescott, Edward C., "A Competitive Theory of Fluctuations and the Feasibility and Desirability of Stabilization Policy," in Stanley Fischer, ed., Rational Expectations and Economic Policy, Chicago: University of Chicago Press, 1980, pp. 169-89.

Marriott, F. H. C. and Pope, J. A., "Bias in the Estimation of Autocorrelations," Biometrika, December 1954, 41, 393-403.

McCallum, Bennett T., "On 'Real' and 'Sticky-Price' Theories of the Business Cycle," Journal of Money, Credit, and Banking, November 1986, 18, 397-414.

Nelson, Charles R. and Plosser, Charles I., "Trends and Random Walks in Macroeconomic Time Series: Some Evidence and Implications," Journal of Monetary Economics, September 1982, 10, 139-62.

Orcutt, Guy H. and Winokur, Herbert S., "First Order Autoregression: Inference, Estimation, and Prediction," Econometrica, January $1969,37,1-14$.

Perron, Pierre, "Trends and Random Walks in Macroeconomic Time Series: Further
Evidence from a New Approach," Journal of Economic Dynamics and Control, June/September 1988, 12, 297-332.

, "The Great Crash, the Oil Price Shock, and the Unit Root Hypothesis," Econometrica, November 1989, 57, 13611402.

and Phillips, Peter C. B., "Does GNP Have a Unit Root? A Re-evaluation," Economics Letters, 1987, 23 (2), 139-45.

Phillips, Peter C. B., "Bayesian Routes and Unit Roots: De Rebus Prioribus Semper Est Disputandum," Journal of Applied Econometrics, October-December 1991, 6, 435-73.

and Perron, Pierre, "Testing for a Unit Root in Time Series Regression," Biometrika, 1988, 75 (2), 335-46.

Rudebusch, Glenn D., "Trends and Random Walks in Macroeconomic Time Series: A Re-examination," International Economic Review, August 1992, 33, 661-80.

Stine, Robert A. and Shaman, Paul, "A Fixed Point Characterization for Bias of $\mathrm{Au}-$ toregressive Estimators," Annals of Statistics, 1989, 17 (3), 1275-84.

Stock, James H., "Confidence Intervals for the Largest Autoregressive Root in U.S. Macroeconomic Time Series," Journal of Monetary Economics, November 1991, 28, 435-59.

and Watson, Mark W., "Does GNP Have a Unit Root?" Economics Letters, 1986, 22 (2), 147-51.

Stulz, René M. and Wasserfallen, Walter, "Macroeconomic Time-Series, Business Cycles, and Macroeconomic Policies," CarnegieRochester Conference Series on Public Policy, Spring 1985, 22, 9-54.

Zhang, Hong-Ching, "Reduction of the Asymptotic Bias of Estimators of Parameters in the Autoregressive and Linear Trend Models," Ph.D. dissertation, Department of Statistics, University of Pennsylvania, 1989. 